\title{
The 2006 hot phase of Romano's star (GR 290) in M 33^
}

\author{
R. F. Viotti ${ }^{1}$, S. Galleti ${ }^{2}$, R. Gualandi ${ }^{2}$, F. Montagni ${ }^{3}$, V. F. Polcaro ${ }^{1}$, C. Rossi ${ }^{3}$, and L. Norci ${ }^{4}$ \\ 1 INAF, Istituto di Astrofisica Spaziale e Fisica Cosmica di Roma, via Fosso del Cavaliere 100, 00133 Roma, Italy \\ e-mail: roberto.viotti@iasf-roma.inaf.it \\ 2 INAF, Bologna Astronomical Observatory, Loiano Observing Station, Loiano, Italy \\ 3 Dipartimento di Fisica, Università di Roma La Sapienza, Pz.le Aldo Moro 3, 00185 Roma, Italy \\ 4 School of Physical Sciences and NCPST, Dublin City University, Glasnevin, Dublin 9, Ireland
}

Received 21 December 2006 / Accepted 28 January 2007

\begin{abstract}
Context. Understanding the nature of the instabilities of LBVs is important to understand the late evolutionary stages of very massive stars.

Aims. We investigate the long term, S Dor-type variability of the luminous blue variable GR 290 (Romano's star) in M 33, and its 2006 minimum phase.

Methods. New spectroscopic and photometric data taken in November and December 2006 were employed in conjunction with already published data on GR 290 to derive the physical structure of GR 290 in different phases and the time scale of the variability. Results. We find that by the end of 2006, GR 290 had reached the deepest visual minimum so far recorded. Its present spectrum resembles closely that of the Of/WN9 stars, and is the hottest so far recorded in this star (and in any LBV as well), while its visual brightness decreased by about $1.4 \mathrm{mag}$.

Conclusions. This first spectroscopic record of GR 290 during a minimum phase confirms that, similarly to AG Car and other LBVs, the star is subject to ample $S$ Dor-type variations, being hotter at minimum, suggesting that the variations take place at constant bolometric luminosity.
\end{abstract}

Key words. stars: evolution - stars: variables: general - stars: individual: GR 290 - stars: Wolf-Rayet - galaxies: individual: M 33 stars: early-type

\section{Introduction and observations}

Romano's star (GR 290) is one of the most luminous hot variable stars in M 33 (Viotti et al. 2006). Its historical light curve is characterised by ample, long-term variations between 16.7 and 18.1 in the $B$-band (Romano 1978; Kurtev et al. 2001). This behaviour is reminiscent of the long-term photometric variations of the S Doradus variable stars that are generally accompanied by colour-index variations, the stars being bluer when fainter in the visual. The spectroscopic monitoring of a number of LBVs confirmed that during minimum they display a hotter spectral type. For instance AG Car changes from a Be/Ae spectral type at maximum visual magnitude, to an Of/WN9 type at minimum (e.g., Caputo \& Viotti 1970; Stahl 1986; Viotti et al. 1993). A similar behaviour was also shown by the Large Magellanic Cloud star R 127 (e.g. Stahl et al. 1983). These observations suggest that the variations might take place at constant bolometric luminosity, implying that the effective stellar radius changes by up to one order of magnitude between visual minimum and maximum.

Since 2003 GR 290 has been frequently observed with the $30 \mathrm{~cm}$ Greve telescope. On November 13, 2006 it was noticed that the star was much fainter than previously recorded, with $V \simeq 18.4$ and $R \simeq 18.5$. Thanks to the availability of a "target of opportunity service" of the INAF Bologna Astronomical Observatory (OAB), it was possible to obtain on November 21 , 2006 new photometric and spectroscopic observations of

* Based on observations collected with the $1.52 \mathrm{~m}$ Cassini telescope of the Bologna Astronomical Observatory.
GR 290 with the $1.52 \mathrm{~m}$ Cassini telescope of the Loiano Observing Station of OAB. These observations confirmed that GR 290 was in a deep minimum phase. Using the photometric standards given in Table 2 of Viotti et al. $(2006)^{1}$ we found the following photometric values: $B=18.46 \pm 0.10$ and $V=$ $18.50 \pm 0.04$.

On the same night we took a low resolution spectrum covering the range $\sim 3600-8800 \AA$ with a dispersion of $3.97 \AA$ per pixel, and an effective resolution of about $17 \AA$. Another spectrum was obtained with the same setup on December 14, 2006. Because of the better seeing conditions a smaller entrance aperture was used so that the effective resolution was of about $13 \AA$, with nearly the same $\mathrm{S} / \mathrm{N}$ ratio as the November spectrum. Both spectra are shown in Fig. 1. The fairly good $\mathrm{S} / \mathrm{N}$ allows to identify many emission lines, mostly of the Balmer series and of neutral helium. Of particular interest is the strong 4640-4713 emission feature that is a blend of N II and N III lines, and of $\mathrm{He}$ II $4686 \AA$ and He I $4713 \AA$. In this spectrum the ionised helium line is quite prominent, with an emission peak reaching $60 \%$ of the $\mathrm{H} \beta$ value. $\mathrm{H} \alpha$ presents asymmetric wings - stronger on the red side of the line - that can be attributed to the [N II] 6548 and $6584 \AA$ emission.

1 The star named $k u-e$ in the table is star "F" of Kurtev et al. (2001) with a slightly different $B$ value of $17.29 \pm 0.10$ than that reported in Table 2 of Viotti et al. The standard star "E" of Kurtev et al. (2001) has the following data: $k u-e: \alpha=01: 35: 04.50 \delta=+30: 42: 53.3, B=$ $17.11 \pm 0.10, V=16.44 \pm 0.07, R=16.05 \pm 0.05, I=15.63 \pm 0.05$. 


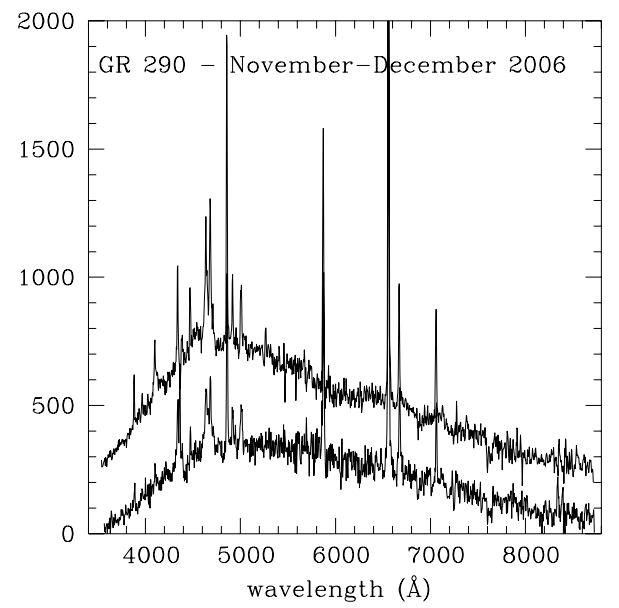

Fig. 1. The November (bottom) and December (top) 2006 low resolution spectra of GR 290. Ordinates are counts with a vertical offset of +200 for the December spectrum. The original November spectrum is slightly smoothed. The long-wavelength side of the $\mathrm{H} \gamma$ emission is affected by the city light line at $4358 \AA$, that is oversubtracted in the December spectrum.

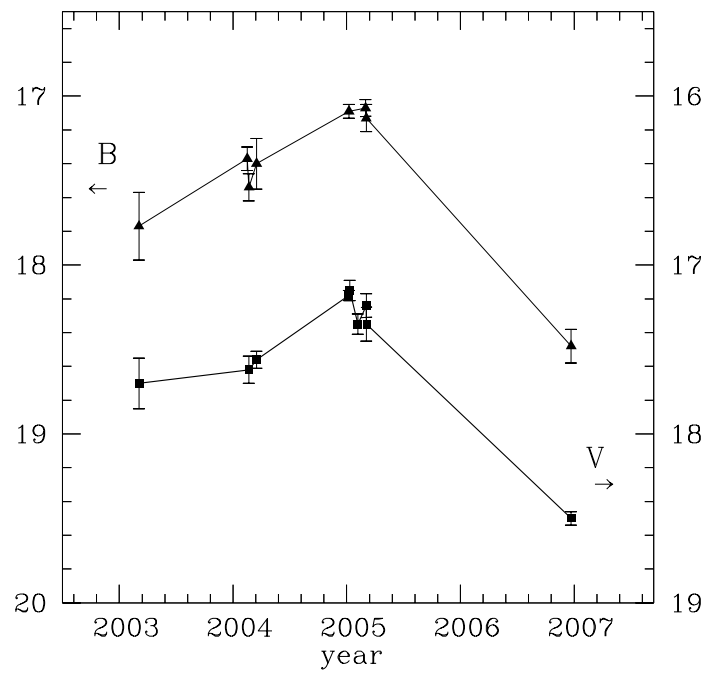

Fig. 2. The recent light curve of GR 290 in the $B$ and $V$ bands (left and right ordinate scales, respectively).

Details on the Loiano and Greve telescopes are given in Viotti et al. (2006).

\section{Discussion}

\subsection{Photometry}

In Fig. 2 we show the recent light curve of GR 290 in the $B$ and $V$ photometric bands. As noticed by Viotti et al. (2006), during 2003-2004 the star gradually brightened by about a factor of $2(-0.7 \mathrm{mag})$ in all $B, V$ and $R$ bands. This brightening phase was followed by a significant bending of the light curve after October 2004, suggesting the beginning of a new descending branch. Our new photometric observations show that the star has become much less bright with a magnitude difference since the end of 2004 of +1.4 both in $B$ and in $V$. This is the deepest minimum of GR 290 in B so far recorded (see Kurtev et al. 2001). Previously, two minima in the blue were recorded in 1961-62 and 1975-77 with $m_{\mathrm{pg}}=17.6-17.7$ (Romano 1978). Because of the poor time coverage of the light curve, other unrecorded

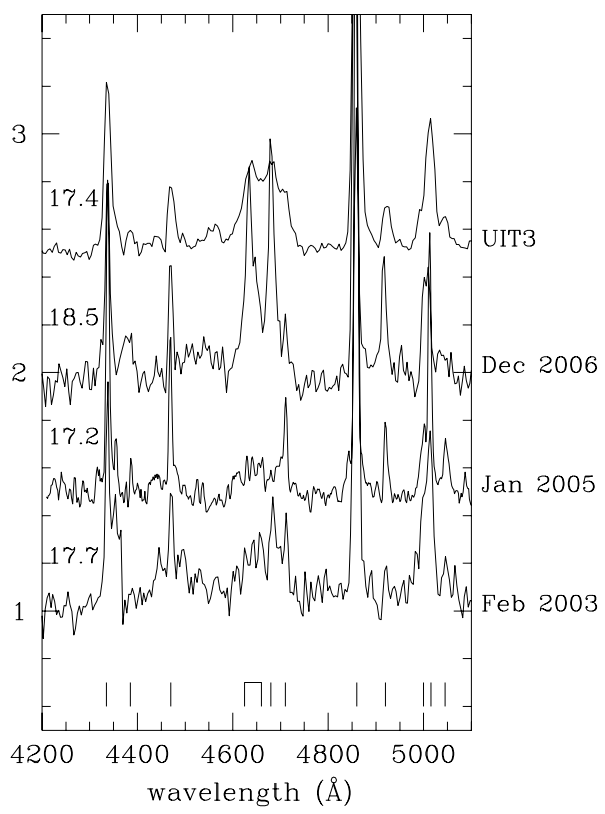

Fig. 3. Spectral variation of GR 290 during 2003-2006. From the bottom: the spectrum of GR 290 on February 2, 2003, January 13, 2005, December 14, 2006, and the spectrum of the Of/WN9 star UIT 3 in M 33 of December 7, 2004. Ordinates are fluxes normalised to the continuum, with vertical offsets. For each spectrum the visual luminosity is also indicated. The vertical bars at the bottom mark the following emission lines: H $\gamma$, HeI $4388 \AA$, HeI $4471 \AA$, the 4630-4650 ̊ feature, HeII $4686 \AA$, HeI $4713 \AA$, H $\beta$, HeI $4922 \AA$, NII 4994-5005 HeI $5016 \AA$, and HeI $5048 \AA$. The peak at $4358 \AA$ is city light.

minima might have occurred during the past half a century. The pseudo-period of a few years and the large amplitude of the recent variation are typical of the S Dor variable stars (e.g. van Genderen 2001).

\subsection{Spectroscopy}

During 2003-2005 many low resolution spectra of GR 290 were collected by us with the Asiago-Cima Ekar and Loiano Telescopes. As discussed by Polcaro et al. (2003) and by Viotti et al. (2006) the spectrum was always characterised by prominent hydrogen and neutral helium emission lines, and weak emission of the 4630-4686 A feature typical of the Of-type stars. Viotti et al. (2006) found that the latter emissions decreased near the 2004 light maximum. The 4200-5100 A range of the Loiano December 2006 spectrum, normalised to the continuum, is compared in Fig. 3. with the spectra taken previously at Loiano with the same instrumental setup. For comparison, the figure also includes the Cima Ekar spectrum of the Ofpe/WN9 star UIT 3 described by Viotti et al. (2006). This spectrum has a slightly lower spectral resolution, so that the 4630-4660 $\AA$ blend, the He II $4686 \AA$ and the He I $4713 \AA$ lines all appear blended. The other spectra of GR 290 taken at Cima Ekar are shown in Fig. 7 of Viotti et al. (2006).

In general the strength of the hydrogen and neutral helium emission lines varied little during 2003-2006 (see also Table 1). The most conspicuous variation is shown by the 4630-4686 $\AA$ emission blend which presently is much brighter than in all the previous spectra. The emission is even stronger than in the M 33 Ofpe/WN9 star UIT 3. The relative strength of the He I and He II lines, and of the 4630-4650 $\AA$ feature can in principle be used for a tentative spectral classification of GR 290 during the 
Table 1. The variable spectrum of GR $290^{1}$.

\begin{tabular}{lcclrrrrr}
\hline \hline Date/target & $V$ & $B-V$ & Sp. type $^{2}$ & $\mathrm{H} \alpha^{3}$ & $\mathrm{H} \beta$ & $4630-86$ & 4713 & 5876 \\
\hline Feb. 02, 2003 & 17.70 & +0.07 & WN10eq & 105 & 26 & 17.3 & 5.4 & 25 \\
Feb. 14, 2004 & 17.56 & -0.16 & WN11eq & 100 & 35 & 2.1 & 2.6 & 24 \\
Dec. 06-07, 2004 & 17.18 & -0.09 & WN11eq & 138 & 29 & 3.9 & 3.1 & 27 \\
Jan. 13, 2005 & 17.36 & -0.11 & WN11eq & 132 & 31 & 9.4 & 4.9 & 19 \\
Nov.-Dec. 2006 & 18.50 & -0.02 & WN9eq & 143 & 32 & $40.7^{4}$ & 2.8 & 32 \\
UIT 3 & & +0.01 & Of/WN9 & 127 & 34 & 31.9 & bl. & 31 \\
\hline
\end{tabular}

Notes. ${ }^{1}$ Equivalent widths of the emission lines in $\AA .{ }^{2}$ Equivalent spectral types for GR $290 .{ }^{3}$ Including the line wings and/or the [N II] lines.

${ }^{4}$ The HeII 4686 A line alone has an emission equivalent width of $18.4 \AA$.

different epochs, although the spectral classification of peculiar, emission line OB stars is still developing, because of the large variety of spectra and the very limited number of members, down to only one representative, for each proposed category.

Using as a reference the Atlas of Peculiar Spectra of OB Stars (Walborn \& Fitzpatrick 2000), we classify our spectra of GR 290 as (Of)WN11 in 2004-2005 when the 4630$4650 \AA$ blend and the He II $4686 \AA$ emission lines were very weak, and (Of)WN10 in February 2003 when the He II line is a little stronger than the He I $4713 \AA$ line. The last NovemberDecember 2006 spectra suggest a spectral type (Of)WN9. The main difference with the spectra of the Atlas is the much stronger Balmer lines in GR 290 (and in UIT 3 as well). This might be due to the greater extension of the emitting atmospheric envelope in these M 33 stars and to the lower optical depth of the hydrogen lines.

Table 1 summarizes the main data on the different phases of GR 290 and reports the equivalent spectral types as discussed above. The $B-V$ colour index does not follow the general photometric trend of the $S$ Dor variable stars, that are bluer when the star is fainter in $V$. However one should take into account that (as discussed by Viotti et al. 2006) in December 2004 GR 290 had a very blue intrinsic (i.e., reddening-corrected) $B-V$ colour of -0.31 . The flux increase of the 4630-4686 $\AA$ emission feature in 2006 might well be accompained by a large deviation of the visual SED from that of a black-body, and by an increase of the colour index, such as that observed in GR 290 in 2003 and 2006. This will require future investigation.

\section{Conclusions}

Viotti et al. (2006) estimated for GR 290 near maximum a bolometric luminosity of $3 \times 10^{6} L_{\odot}$, which places the star among the brightest LBVs. The 2003-2006 photometric and spectroscopic monitoring confirmed that the variations are typical of the $S$ Doradus variable stars, with the star being hotter when fainter in the visible. This fact is better illustrated in Fig. 4 where we show the variation of the $W_{\text {eq }}$ of the 4630-4686 $\AA$ blend as a function of the visual magnitude. The strength of the blend is linked to the stellar flux in the far ultraviolet, while the visual magnitude is related to the effective radius and temperature of the star. The apparent counter-correlation between visual luminosity and temperature suggests that, as in the best studied S Dor variable stars, the variations in GR 290 take place at constant bolometric luminosity. If this is the case, the star should have increased its effective radius during 2003-2004, and have reached a maximum radius (and minimum effective temperature) by the end of 2004. Since the beginning of 2005 GR 290 has inverted the trend and its effective radius decreased (by about a factor

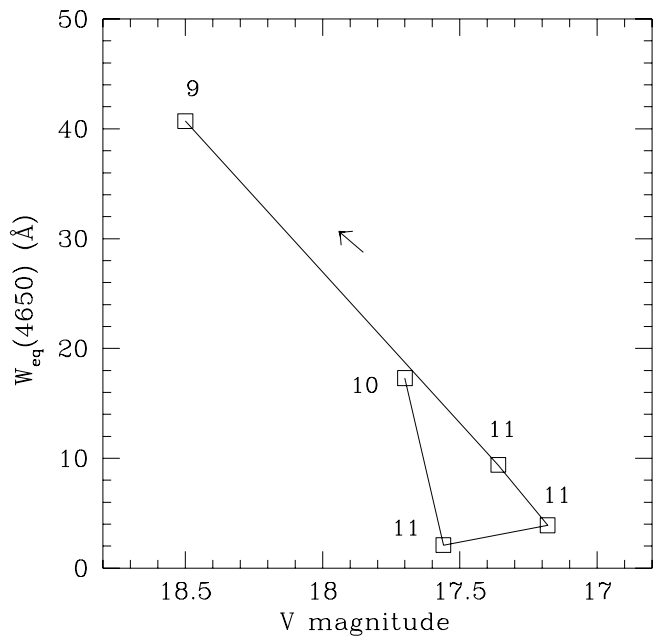

Fig. 4. Equivalent width of the 4630-4686 $\AA$ emission blend as a function of the visual magnitude of GR 290 from February 2003 to December 2006. The arrow indicates the direction of the evolution with time. The labels give the proposed $\mathrm{WN}$-subtypes.

two), while the effective (or Zanstra) temperature has become higher.

Of great interest is the fact that GR 290 presently displays the hottest spectrum ever seen in an LBV. It would be important to understand whether this result is fortuituous, because of the poor spectroscopic monitoring of the other LBVs, or whether it is related to the intrinsic nature of GR 290, which probably is one of the most massive LBVs.

The similarity of the present spectrum of GR 290 with that of the Of/WN9 stars confirms the link between LBVs and this category of star, a fact that has to be taken into consideration in evolutionary models of very massive stars.

Valuable information can be collected on this most interesting category of stars by continuous monitoring, even with medium and small size telescopes.

\section{References}

Caputo, F., \& Viotti, R. 1970, A\&A, 7, 266

van Genderen, A. M. 2001, A\&A, 366, 508

Kurtev, R., Shoulukhova, O., Borissova, J., \& Georgiev, L. 2001, Rev. Mex. Astron. Astrofis., 37, 57

Polcaro, V. F., Gualandi, R., Norci, L., Rossi, C., \& Viotti, R. F. 2003, A\&A, 411,193

Romano, G. 1978, A\&A, 67, 291

Stahl, O. 1986, A\&A, 164, 321

Stahl, O., Wolf, B., Klare, G., et al. 1983, A\&A, 127, 49

Viotti, R., Polcaro, V. F., \& Rossi, C. 1993, A\&A, 276, 432

Viotti, R., Rossi, C., Polcaro, V. F., et al. 2006, A\&A, 458, 225

Walborn, N. R., \& Fitzpatrick, E. L. 2000, PASP, 112, 50 